\title{
A Graph-based Iterative Algorithm for Inverse Problems with Local and
}

\section{Non-local Regularizations}

Ming Chen ${ }^{1, a}$, Chuan Xie ${ }^{2, b}$, Yongchao Wang ${ }^{1, c}$, Zhijie Lin $^{1, d}$, Lei Zhao ${ }^{1, e}$

1The department of computer science and technology, Zhejiang University, China.

${ }^{2}$ Hangzhou vocational and technical college,China.

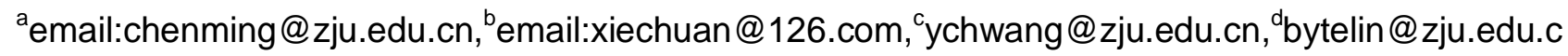
n, eemail:cszhl@zju.edu.cn

Keywords: Inverse Problems, Total Variation, Image Prior

Abstract. I In this paper, we propose a new form of prior which combines the local total variation regularization with non-local graph-based variation regularization to solve such inverse problems in the field of image processing. The algorithm iteratively updates the image and the weight graph calculated from the present image..

\section{Introduction}

The most common form of a prior knowledge is the well-known smoothness based prior [2] which assumes that the image contents generally do not change abruptly in a neighborhood of space. Each pixel in the image is constrained to be relevant to its neighboring pixels. It is a generic image contextual constrain. These methods considering the image smoothness take advantage of the local structure of an image, leaving out the overall structure information of the image. Some non-local methods $[3,4,5]$ thus have been widely studied. The non-local method assumes that some feature of an image does always repeat in different positions in the image. These methods always seek the similar image patches or exemplars meeting some specified measurements in the whole image space, which means they utilize the information of the entire image.

In this paper, we propose a new form of prior for solving general inverse problems in image processing which combines the local total variation regularization with graph-based non-local variation regularization. This graph depicts the relationship among different image patches. This prior makes use of both local and non-local image information to get a better result. Because of the existing of non-local graph-based variation regularization, the problem to be solved in this paper turns to be non-convex. We, therefore, propose an iterative algorithm to work out the inverse problem using such a form of prior, which iteratively updates the image and the weight graph calculated from the current image till convergence. We also show the results when using this new framework in image denoising, image inpainting and image deblurring applications.

\section{The Proposed Algorithm}

In this section, we delve into the iterative algorithm proposed to solve the inverse problem depicted above with the mixed variation regularizations. Formally, the solution to the inverse problem in image processing with local total variation and non-local graph-based variation can be written as follows: 


$$
\tilde{\mathbf{x}}=\underset{\mathbf{x} \in R^{m \times n}}{\arg \min } \frac{1}{2}\|\mathbf{y}-\mathbf{A x}\|^{2}+\lambda_{1} T V(\mathbf{x})+\lambda_{2} \operatorname{NTV}(\mathbf{x})
$$

where $T V(\mathbf{x})$ and $N T V(\mathbf{x})$ are the local and graph-based non-local variation

regularizations respectively. $\lambda_{1}$ and $\lambda_{2}$ are the regularization parameters that need to be adapted according to the amplitude of the noise $\mathbf{n}$, which might be an important and difficult task in practical situations.

An inverse problem with local total variation regularization only is convex, though sometimes may be non-differential. There has been plenty of researches about how to solve such a TV-based problem [18], [16]. However, the introduction of graph-based non-local regularization term makes the problem (3) non-convex.

To solve such an non-convex ill-posed problem, we propose an iterative algorithm which iteratively updates the image and the weight graph calculated from the current image till convergence. Concretely, the whole algorithm can be divided into two subproblems. The first subproblem is building the weight graph based on the current image content. And the second is to solve the problem with mixed regularization terms. Here, the non-local total variation is obtained from the weight graph calculated before, in which the vertexes are the pixels in the image and the edges denote the weights between different pixels. Such an algorithm frame, to some extent, is akin to the widely known EM algorithm [19].

To solve the inverse problem discussed above, we use an iterative optimization algorithm which can be divided into two subprocesses, building the weight graph according to definition in (8) and solving the problem defined by (10) and (11). More specifically, we first initialize an image $\mathbf{x}$ with its pixel values sampled from a normal distribution. And then the weight graph corresponding to $\mathbf{x}$ will be built according to (8). Now we can establish the objective function defined in (10) by utilizing image data and the weight graph. Next we solve the optimization problem in (11) using some gradient descend methods with the following gradient:

In our approach, L-BFGS-B [11] is chosen to solve the optimization problem, which is freely available and reasonable for dealing with high-dimensional optimization problem. L-BFGS-B is an extension of the L-BFGS algorithm [32]. The B here stands for the inclusion of Boundaries.The whole procedure of the proposed iterative optimization algorithm is formally stated below:

1) Initialization: Sample $\mathbf{x}^{0}$ from $N(0,1)$ and set $k \leftarrow 0$.

2) Build the weight graph: Compute the weight graph $G^{(k)}=G(w)$ with $w$ calculated via equation (7).

3) Establish the objective function: Calculate $f\left(\mathbf{x}^{k}\right)$ leveraging equation (10).

4) Compute the gradient matrix: Update the image contents using L-BFGS-B with 


$$
\frac{\partial f\left(\mathbf{x}^{(k)}\right)}{\partial \mathbf{x}_{i, j}^{k}}=-\mathbf{A}^{T}\left(\mathbf{y}^{(k)}-\mathbf{A} \mathbf{x}^{(k)}\right)_{i, j}+\lambda_{1} \frac{\partial T V\left(\mathbf{x}^{(k)}\right)}{\partial \mathbf{x}_{i, j}^{(k)}}+\lambda_{2} \frac{\partial N T V\left(\mathbf{x}^{(k)}\right)}{\partial \mathbf{x}_{i, j}^{k}}
$$

Denote the result as $\mathbf{x}_{l}$.

5) Stopping criterion: While not converged, set $k \leftarrow k+1, \mathbf{x}^{(k)}=\mathbf{x}_{l}$ and go back to 2 .

\section{Acknowledgements}

This work was financially supported by the Zhejiang Province Natural Science Foundation (Y16F020023), Zhejiang Province Project" Key technology research and system development of image restoration based on convolutional neural network" and Zhejiang Province Project" Research on the key technology and system development of automatic generation of video virtual viewpoint for security monitoring".

\section{References}

[1] M. Bertero, P. Boccacci, Introduction to inverse problems in imaging, CRC press, 1998.

[2] M. Bertero, T. A. Poggio, V. Torre, Ill-posed problems in early vision, Proceedings of the IEEE 76 (8) (1988) 869-889.

[3] A. Buades, B. Coll, J.-M. Morel, A non-local algorithm for image denoising, in: 2005 IEEE Computer Society Conference on Computer Vision and Pattern Recognition (CVPR'05), Vol. 2, IEEE, 2005, pp. 60-65.

[4] P. Arias, V. Caselles, G. Sapiro, A variational framework for non-local image inpainting, in: International Workshop on Energy Minimization Methods in Computer Vision and Pattern Recognition, Springer, 2009, pp. 345-358.

[5] A. Dauwe, B. Goossens, H. Q. Luong, W. Philips, A fast non-local image denoising algorithm, in: Electronic Imaging 2008, International Society for Optics and Photonics, 2008, pp. 681210-681210.

[6] L. I. Rudin, S. Osher, E. Fatemi, Nonlinear total variation based noise removal algorithms, Physica D: Nonlinear Phenomena 60 (1) (1992) 259-268.

[7] T. Chan, J. Shen, Local inpainting models and tv inpainting, SIAM J. Appl. Math 62 (3) (2001) 1019-1043.

[8] A. Levin, A. Zomet, Y. Weiss, Learning how to inpaint from global image statistics, in: Computer Vision, 2003. Proceedings. Ninth IEEE International Conference on, IEEE, 2003, pp. 305-312.

[9] T. Chan, S. Esedoglu, F. Park, A. Yip, Recent developments in total variation image restoration, Mathematical Models of Computer Vision 17 (2).

[10] M. V. Afonso, J. M. Bioucas-Dias, M. A. Figueiredo, An augmented lagrangian approach to the constrained optimization formulation of imaging inverse problems, Image 
Processing, IEEE Transactions on 20 (3) (2011) 681-695.

[11] J. P. Oliveira, J. M. Bioucas-Dias, M. A. Figueiredo, Adaptive total variation image deblurring: a majorization-minimization approach, Signal Processing 89 (9) (2009) 1683-1693.

[12] J. M. Bioucas-Dias, M. A. Figueiredo, J. P. Oliveira, Total variation-based image deconvolution: a majorization-minimization approach, in: 2006 IEEE International Conference on Acoustics Speech and Signal Processing Proceedings, Vol. 2, IEEE, 2006, pp. II-II.

[13] S. D. Babacan, R. Molina, A. K. Katsaggelos, Total variation super resolution using a variational approach, in: 2008 15th IEEE International Conference on Image Processing, IEEE, 2008, pp. 641-644.

[14] A. Marquina, S. J. Osher, Image super-resolution by tv-regularization and bregman iteration, Journal of Scientific Computing 37 (3) (2008) 367-382.

[15] M. Lysaker, X.-C. Tai, Iterative image restoration combining total variation minimization and a second-order functional, International journal of computer vision 66 (1) (2006) $5-18$.

[16] A. Beck, M. Teboulle, Fast gradient-based algorithms for constrained total variation image denoising and deblurring problems, IEEE Transactions on 